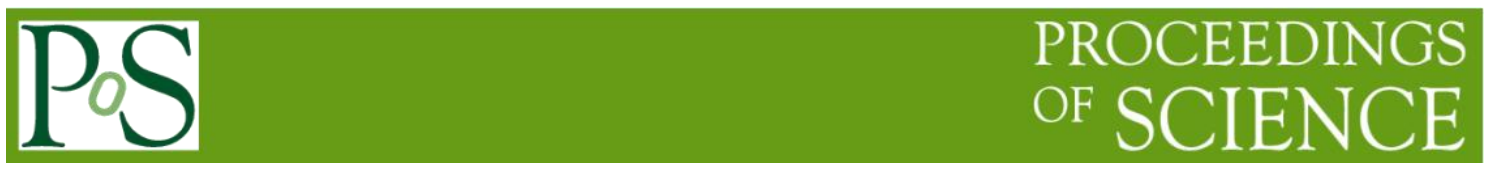

\title{
QuarkNet Coordination of a Cosmic Ray Experiment Outreach Project During a Total Solar Eclipse
}

\author{
Mark Adams ${ }^{1}$ \\ University of Illinois at Chicago \\ Physics Department, 845 W. Taylor Street, Chicago, IL 60607, USA \\ E-mail: adams@uic.edu
}

Nathan Unterman, Clarissa Carr, Jacob Rosenberg, Anthony Valsamis

Glenbrook North High School

Tamar Dallal, Michelle Matten, Jacob Miller, Ezra Schur, Allen Sears

Ida Crown Jewish Academy

\begin{abstract}
:
The QuarkNet program has distributed hundreds of cosmic ray detectors for use in high schools and research facilities throughout the world over the last two decades. To test the hypothesis that the rate of cosmic rays may change during a solar eclipse, a collaboration of high school students and teachers came together to build cosmic ray telescopes that were reproduced by thirty groups across the USA. Groups from a wide geographic region collected data and measured rates of muons during the solar eclipse on August 21, 2017. The process of outreach and execution will be discussed. No variation in the muon flux during the eclipse was observed.
\end{abstract}

36th International Cosmic Ray Conference -ICRC2019-

July 24th - August 1st, 2019

Madison, WI, U.S.A.

\section{${ }^{1}$ Speaker}




\section{Introduction}

QuarkNet is a mature teacher development program under which fifty high energy physics research groups in the USA have developed long-term collaborations with high schools in their vicinity. Schools take part in activities with access to data from the experiments at the Large Hadron Collider and the neutrino program at Fermilab. In addition, many schools have also been provided with a cosmic ray detector with which they develop their own experiments utilizing muons.

A group of teachers and students in the QuarkNet Center at the University of Illinois at Chicago designed a measurement of cosmic ray muons to take place during the 2017 total solar eclipse that covered most of the continental United States. They used the standard cosmic ray detector ${ }^{1}$ available to all QuarkNet centers and developed a protocol and hardware configuration that allowed users to record the flux of cosmic rays in the direction of the eclipse, using muons. Their designs were provided to all centers. Over fifty sites collected data during the eclipse, even though the eclipse took place during summer break.

The total solar eclipse of August 21, 2017 provided the opportunity to measure the cosmic ray flux at the surface for muons across the continental United States. Other groups have reported on low energy gamma ray showers ${ }^{2}$. This measurement is the first with cosmic ray muons detected by scintillation counters. Although the short period of the eclipse combined with the small angular sizes of the sun and moon does not allow a measurement of the shadow that either object casts in the cosmic ray flux ${ }^{3}$, we hypothesized that the eclipse could indirectly affect a larger region of the sky. Although day/night rate comparisons show that the sun is not a significant source of cosmic rays, the QuarkNet teachers leaped at the opportunity to firmly establish an upper limit of muon flux from the sun during the eclipse.

\section{Design}

The teachers' muon flux idea germinated eight months before the eclipse. Prototypes were built to determine the best compromise between muon rate and angular acceptance. Teams designed detector stands using components that were available in hardware stores throughout the USA, while keeping the costs below a hundred dollars (US). Two designs were provided to other QuarkNet centers: a tracking detector to follow the sun, with angular acceptance of 3-10 degrees, to be mounted on a borrowed telescope mount; and a fixed-angle design that measured the muon flux as the sun passed across its acceptance. Both designs used QuarkNet detectors containing four $25 \mathrm{~cm} \times 30 \mathrm{~cm}$ scintillation counters, GPS time stamp, and data acquisition card. Counter-pairs overlapped each other, and the pairs were separated by either $3.3 \mathrm{~m}$ (tracking) or $2 \mathrm{~m}$ (fixed) as shown in Figure 1. The fixed-angle detectors had an angular acceptance in the direction parallel to the path of the sun ( 20 degrees) larger than in the perpendicular direction. Several tracking detectors collected data with their large acceptance ( 10 degrees) oriented either along the path of the sun (perpendicular to the earth's magnetic field) or along the field lines, because the shape or angular extent of any effect was unknown. 


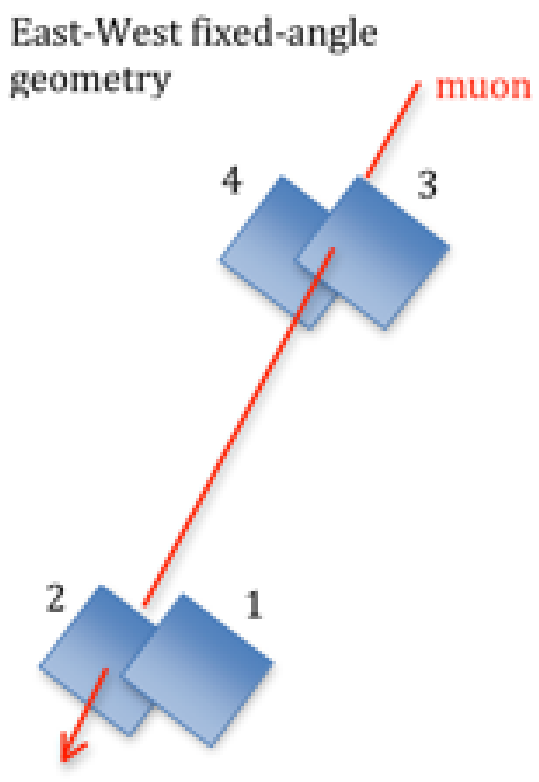

Figure 1. Side view of detector configuration for the fixed-angle telescope.

Many design challenges were addressed: muon trigger rates versus pointing resolution; size of overlap of counter pairs which generate high resolution sun-pointing combinations; nearneighbor pairs monitoring almost the entire sky to allow normalization to avoid corrections due to atmospheric pressure changes; alternative normalization based on whole-sky muon acceptance from independent, vertically stacked detectors; support frame construction; triggering logic operation (2-fold coincidence); alignment schemes; and background estimates and subtraction techniques. A website was created to organize collaborators, to provide construction instructions and data taking procedures, and to share results. All data was uploaded to the QuarkNet e-Lab (i2u2.org) where analysis was carried out. Tools were developed to measure rates from various combination of scintillation counters every ten minutes.

Cosmic Ray rates in the direction of the sun were measured during the solar eclipse at locations throughout the USA. Data was collected at school locations in both total and partial eclipse regions. Stacked detectors located around the world collected data in case there was a global effect, i.e. not limited to directions near the sun or even confined to the regions of the eclipse.

The eclipse lasted approximately 2 minutes. Typical expected muons rates for each detector type, presented in Table 1, demonstrate the compromises made in the detector design. The fixedangle detector had large count rates but small signal to noise, as the sun moved across its large acceptance region. An advantage was that background rates from the empty sky remain constant and are simple to subtract. The tracking detector operated in two modes: a 10-degree acceptance with 5\% statistical error and a smaller 3-degree region with 30\% expected errors. It constantly monitored the region around the sun, however since its zenith angle changed, background subtraction from the empty sky was more complex. 


\begin{tabular}{|l|c|c|}
\hline \multicolumn{1}{|c|}{ Detector type } & $\begin{array}{c}\text { Muon Rate } \\
\text { (counts per 10 minutes) }\end{array}$ & $\begin{array}{c}\text { Angular Acceptance } \\
\text { (degrees) }\end{array}$ \\
\hline Stack & 3000 & 150 \\
\hline Fixed-Angle & 150 & 20 \\
\hline Tracker & 50 & 10 \\
\hline Tracker (4-fold trigger) & 3 & 3 \\
\hline Sun and moon angular size & 0.1 & 0.5 \\
\hline
\end{tabular}

Table 1. Typical muon counts in 10-minute bins for detectors with various angular acceptances.

\section{Background studies and preparation for eclipse day}

The UIC QuarkNet group distributed their designs throughout QuarkNet and tested final prototypes and analysis techniques during a 5-day summer workshop. They travelled to a region of total eclipse three days before the event to make final modifications and to obtain calibration data. Four detectors were assembled: one fixed-angle; two tracking telescopes; and a vertical stack. The goal of this project was to measure the fraction of cosmic ray muons that come from the sun. Several backgrounds were measured.

The contamination of multi-muon triggers in the tracking telescope was measured using time of flight between counters to be less than $3 \%$ of the rate of muons in the direction of the sun.

Even though the muon-shadows of the moon and sun were estimated to be negligible, data was obtained on the days preceding the eclipse using four runs: with only the sun in the acceptance, only the moon in the acceptance, both in the acceptance, and empty sky.

The alignment of tracking detector towards the sun was a multi-step process. It relied on aligning targets mounted on the telescope using their shadows caused by the sun, collecting data on sunny days before the eclipse, and using a laser to transfer a the position vs time to the ground, so that the telescope's path could be reproduced on eclipse day even if sun was not visible.

Several problems were encountered and solved. Not all counters operated stably. Shielding required to reduce light leaks in the counters from direct sunlight generated intense heat that melted support tape and caused intermittent connections in some low voltage cables. We were prepared with spare cables. Redundant counters allowed off-line rejection of troubling counters if discovered later. One of the tracking telescope mounts collapsed on the first day and had to be repaired. The commissioning and calibration runs were essential to the success of the project.

Two normalization schemes to avoid correcting the muon flux changes due to atmospheric pressure over time: the nearest-neighbor pair triggers (12 and 34 on figure 1) on the telescopes sensitive to muons from most of the sky; and independent, vertically stacked detectors.

\section{Results}

Details of analysis of data from a fixed-angle telescope in Nashville, TN and a tracking telescope located at Hillsborough, MO are discussed below. The period of the eclipse was defined as 90 seconds. Backgrounds were obtained from times outside that period.

\subsection{Fixed-Angle telescope results from Nashville data}


The ratio of rates of muons parallel to sun (counters 1-3) to the rate of muons from the full sky (counters 3-4) versus time in 10-minute bins over a 2-day period is shown in Figure 2. No change in ratio is observed during the eclipse (near bin 175).

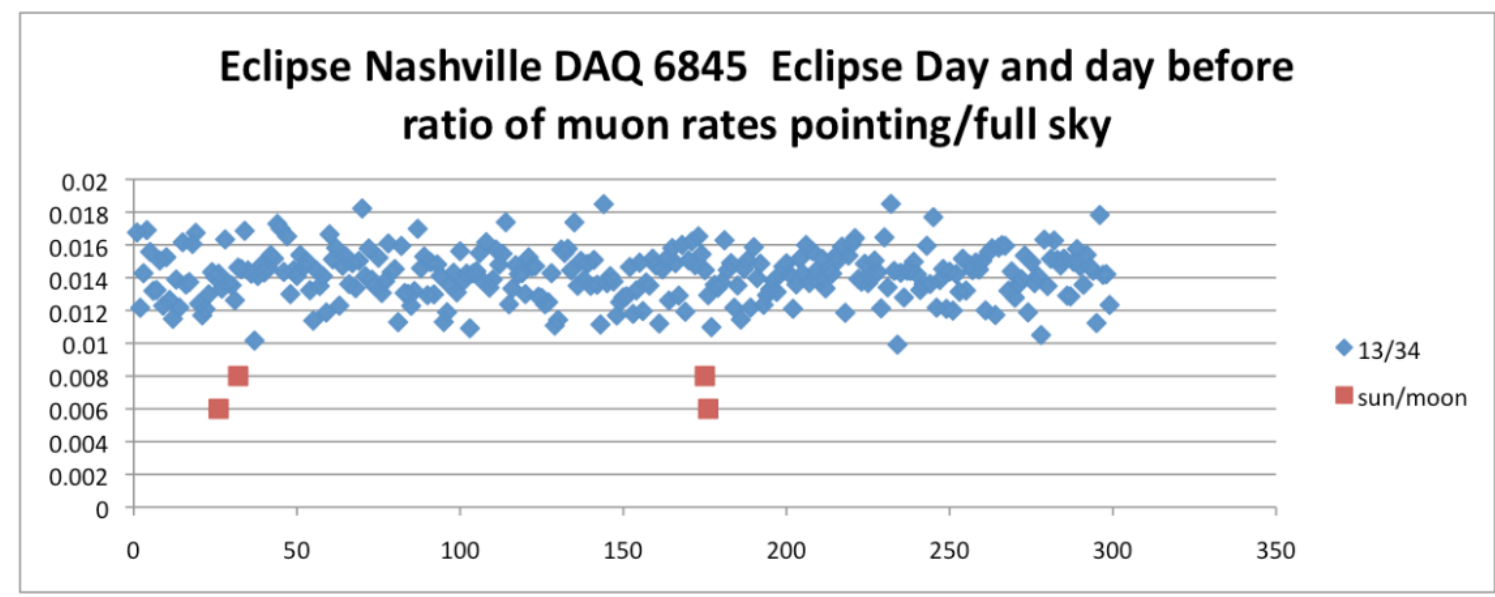

Figure 2. Rate of muons in the direction of the sun divided by the rate of muons from the full sky versus time in 10-minute bins, using a fixed-angle detector. Positions of the sun and moon at local noon are also indicated.

\begin{tabular}{|l|c|}
\hline Condition & Ratio pointing to sun/full sky (\%) \\
\hline Empty Sky & $1.42+-0.01$ \\
\hline Moon only & $1.38+-0.05$ \\
\hline Sun only & $1.44+-0.05$ \\
\hline Eclipse & $1.42+-0.05$ \\
\hline
\end{tabular}

Table 2. Average of the rate of muons in the direction of the sun divided by the rate of muons from the full sky for the data collected in Nashville.

The average of the ratio of muons rates for four conditions are presented in Table 2. Ignoring the moon and sun muon shadow data; combining similar data from five sites across the USA yields for the ratio of muon rates from the eclipse compared to empty sky background a value of $\mathrm{R}=0.999+-0.018$. The $95 \%$ confidence level limit for the fraction of muons detected at the surface, attributed to the sun, is less than $5 \times 10^{-5}$.

\subsection{Tracker telescope results from Hillsborough data}

Results from the tracking telescope technique also showed no variation during the eclipse. Normalization proved more difficult because the muon rate depends strongly on the zenith angle, however, no significant difference in the muon rate during the eclipse was observed using this technique.

Rates along the telescope axis under conditions: eclipse; moon only in the sky; sun only in the sky; empty sky are shown in Figure 3. Errors are shown only for the eclipse data, but all others are similar. Most backgrounds were collected on days before the eclipse at the eclipse location during times when the moon or sun's trajectory in zenith angle matched that of the sun's during the eclipse. Additional, higher statistics samples were obtained later at a different latitude for the moon-only and empty sky samples. The moon's path was required to be similar to the sun's path 
on eclipse day and alignment of the telescope was obtained with the light from a full moon. No large variations between samples were observed.

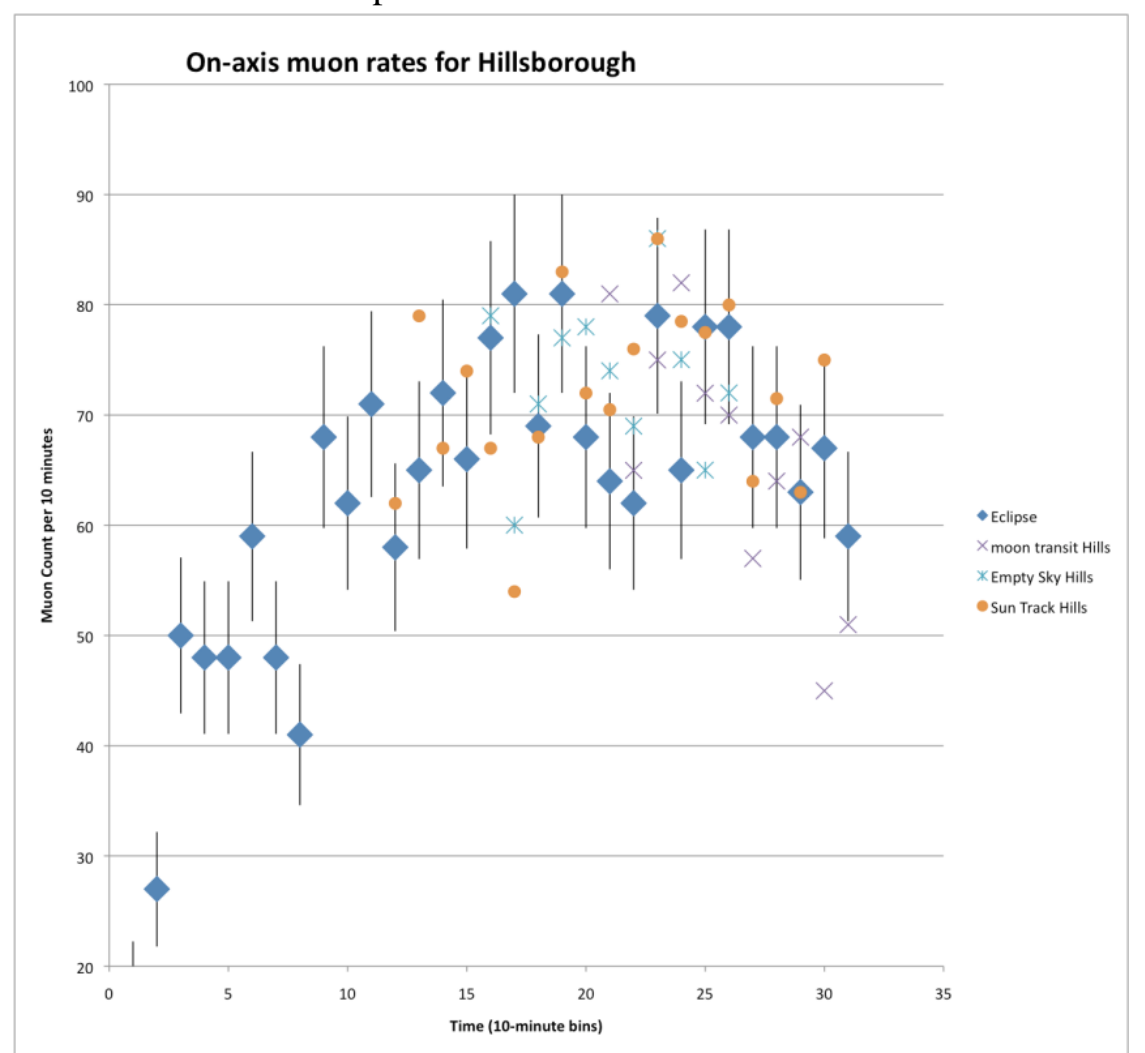

Figure 3. Muon counts versus time in 10-second bins for the tracking telescope in Hillsborough. The maximum of the eclipse is found in bin 22 .

Figure 4 contains the additional calibrations performed at other locations later in the fall. Rates are also scaled by $\cos ^{2}(\theta)$ to account for the azimuthal rate dependence. Empty sky was collected over many days, so their errors are shown. Normalization of rates due to atmospheric pressure changes is performed by referring to the rate in a nearby vertical stacked detector that remained constant during all testing periods. $\cos (\theta)$ scaling is not valid over entire range of zenith angles. Because of the remaining large uncertainties due to varying zenith angle, no limit has been extracted. Under the assumption of no sun or moon shadow effect, the muon flux after $\cos (\theta)$ scaling is compared for the eclipse and empty sky samples $R_{\text {eclipse }} / R_{\text {empty }}=0.998$ with $4 \%$ errors. 


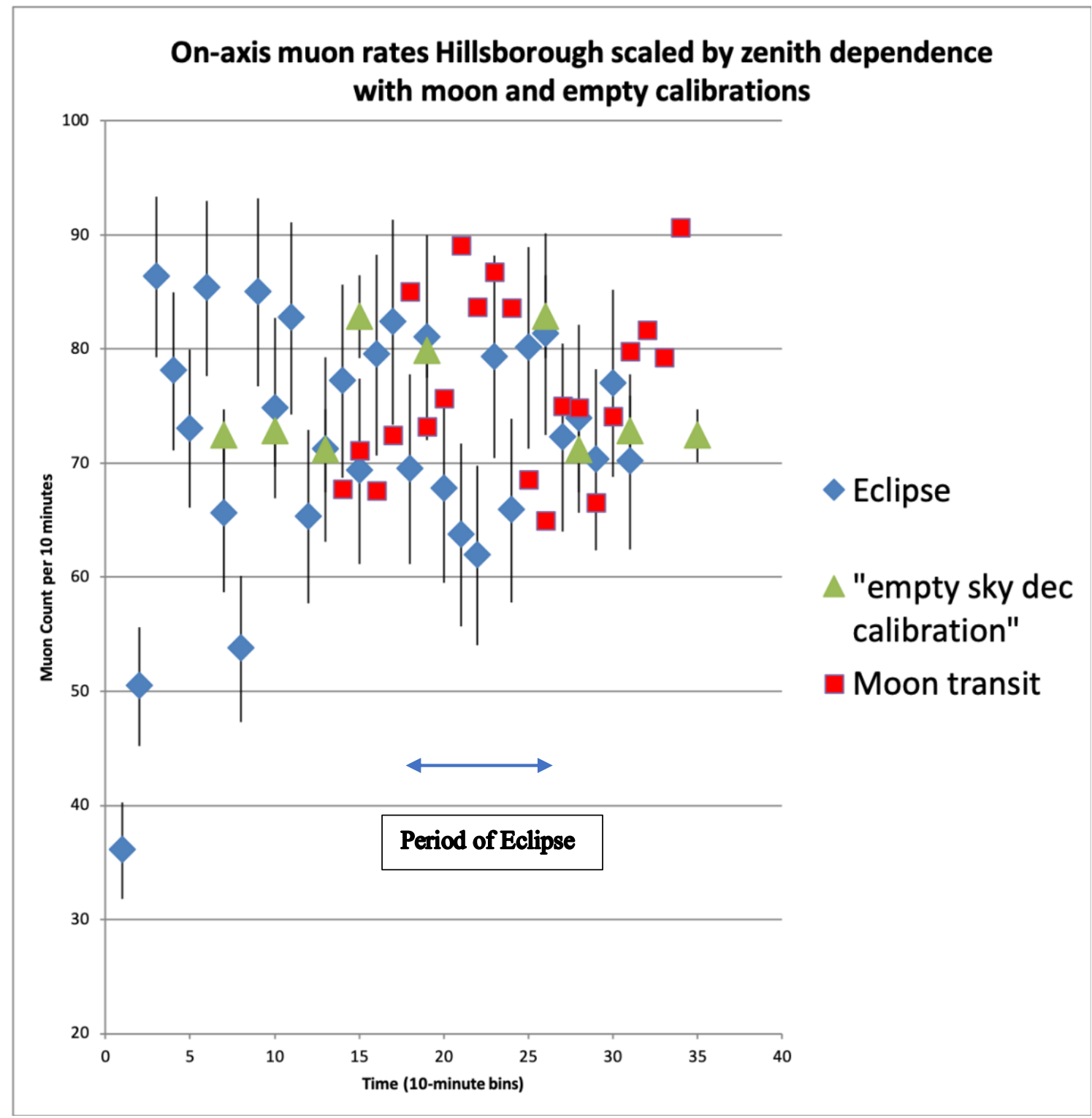

Figure 4. Muon counts scaled by $\cos ^{2}(\theta)$, where $\theta$ is the zenith angle, for the tracking telescope in Hillsborough. The maximum of the eclipse is found in bin 22 .

\section{Conclusions}

High school teachers from the QuarkNet program throughout the USA (see Figure 5) formed a collaboration to measure the cosmic ray muon flux during the 2017 total solar eclipse. No statistically significant changes in the muon rate in the direction of the sun were observed during the eclipse. In addition, using vertical telescopes, no rate differences were observed in regions throughout the world, whether or not they experienced the eclipse. Two telescope techniques were used. Results from fixed-angle telescope at five sites were used to report a 95\% confidence level of $5.0 \times 10^{-5}$ on the fraction of muons detected on the earth's surface due to the sun. Additional data collected throughout the USA can be used to improve this limit. 


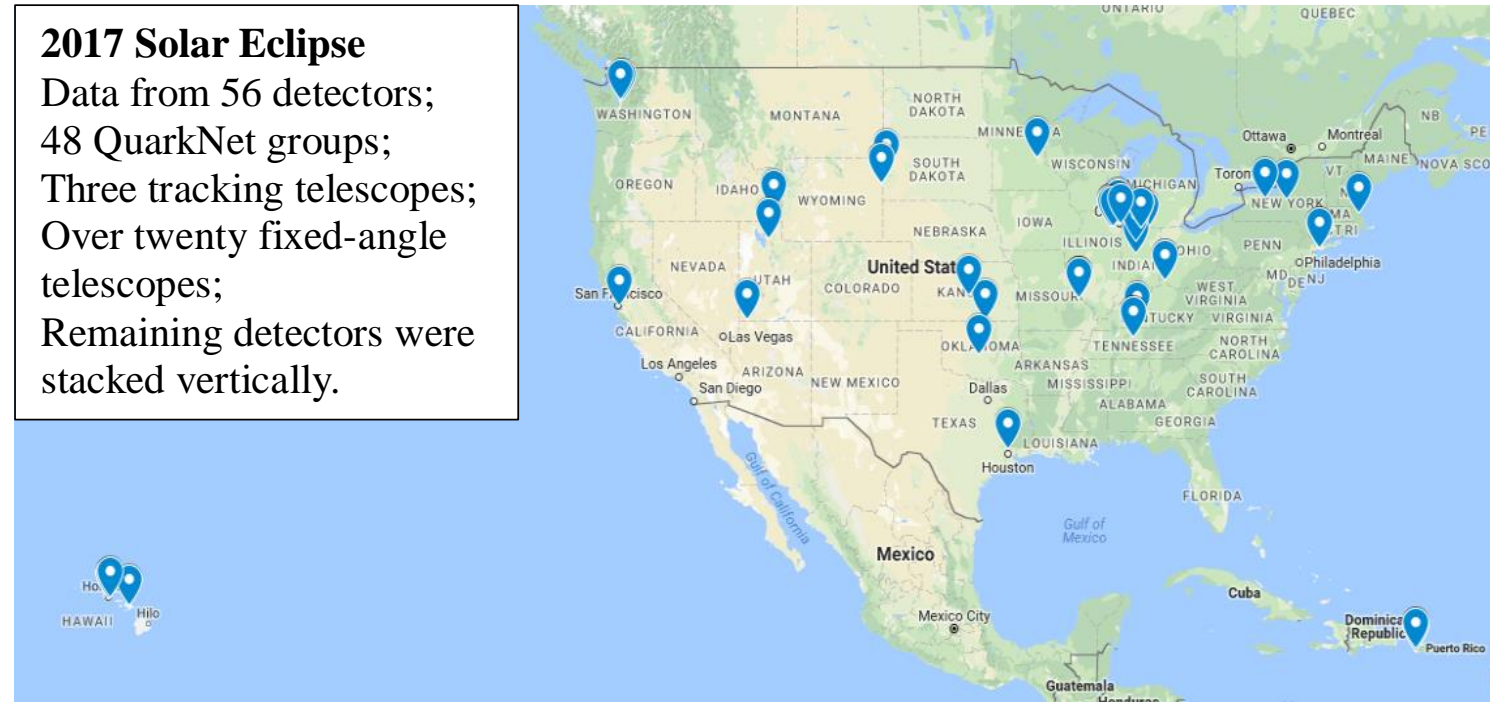

Figure 5: Map of active QuarkNet cosmic ray detectors in the USA during the 2017 solar eclipse.

6. Acknowledgements

The 2017 Cosmic Ray Eclipse project was supported by QuarkNet, the National Science Foundation and Fermilab. We wish to thank Jefferson College in Hillsborough, MO for hosting the UIC QuarkNet team during our three-days of data taking.

\section{References}

[1] S. Hansen, et. al., Low Cost Data Acquisition Card for School-Network Cosmic Ray Detectors. [arxiv.org/pdf/physics/0311060].

[2] Kandemir,G., Guclu, M.C., Geckinli,M, Firat, C. Boydag,S., Ozgguc, A., Variation of Cosmic Ray Intensity During a Solar Eclispe, August 11, 1999, ASP Conference Series, Vol. 205, 2000.

Bhaskar,A., Purohit,A., Hemalatha,M., Pai,C., Raghav,A., Gurada,C., Rahda,S., Yadav,V., Desai,V., Chitnis,A., Sarpotdar,P., Patankar,A., A study of secondary cosmic ray flux variations during the annular eclipse of 15 January 2010 at Ramaeswaram, India, AstroParticle Physics 35(5):223. 2011.

[3] The sun's muon shadow was found, during a previous experiment by this QuarkNet group, to be consistent with a $0.8 \%$ decrease in the muon flux around local noon. 